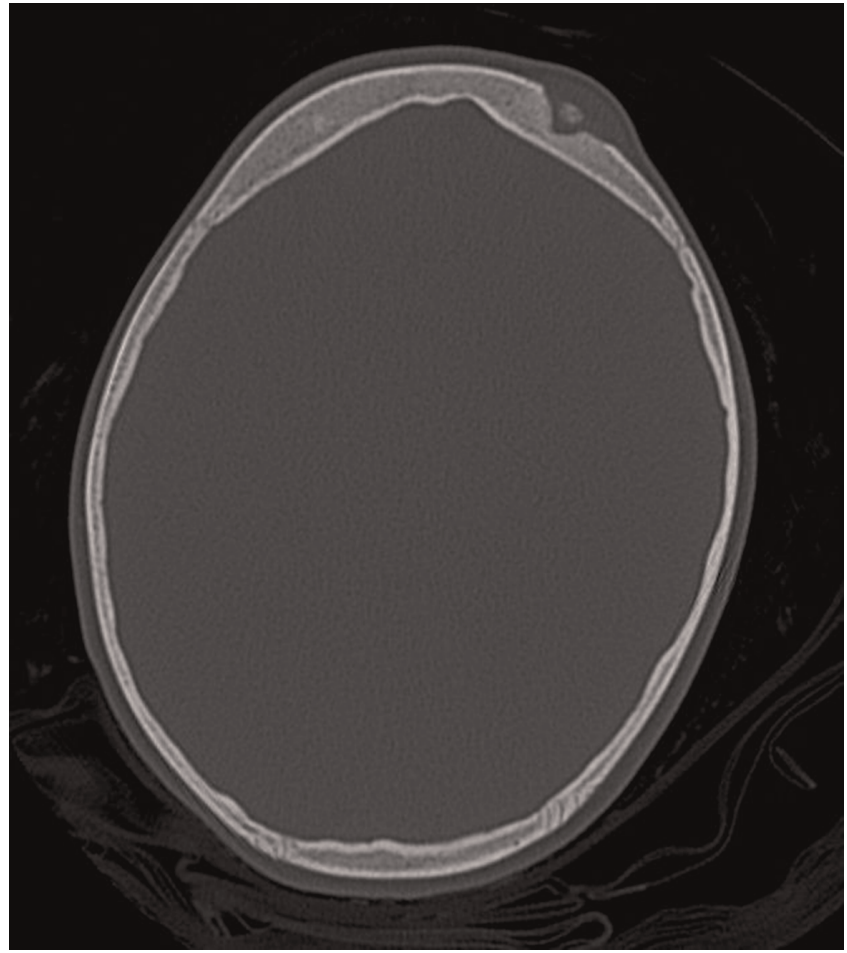

Abstract P0-0228 Figure 1

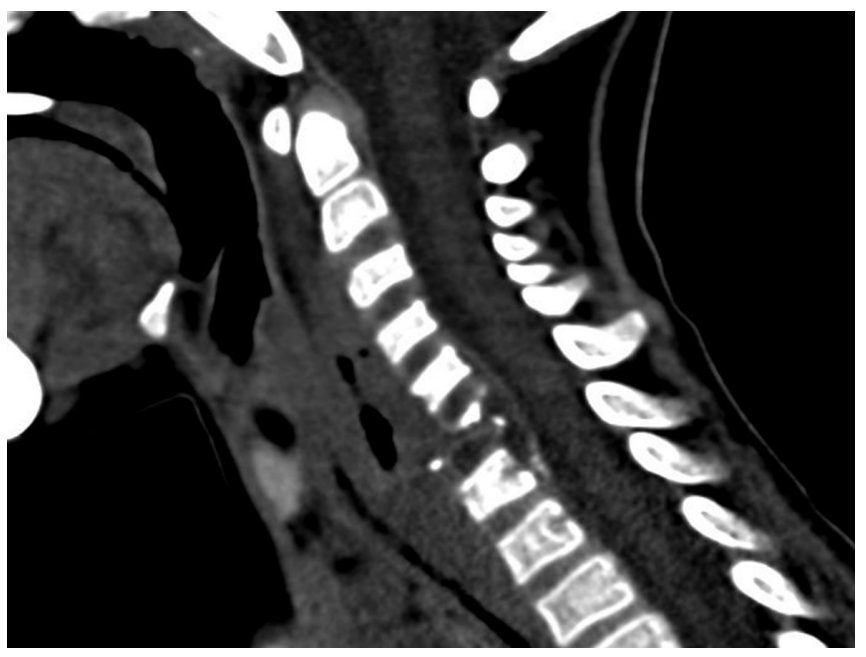

Abstract P0-0228 Figure 2

Conclusions There should be higher access to information about RVGE and vaccination against it. Breastfeeding till age of 2 years may facilitate severity of RVGE.

Part of the study "Clinical peculiarities of rotaviral infection, molecular epidemiology and health associated life quality for hospitalised children and their family members", financially supported by Riga Stradins University.

\section{PO-0228 ATYPICAL PRESENTATION OF TUBERCULOSIS IN A PATIENT WITHOUT BCG VACCINE}

1] Correa de Lacerda, ${ }^{1}$ PLF Perinei, ${ }^{1}$ AGN Reis, 'DAG Ribeiro, ${ }^{1} \mathrm{CHS}$ Vasques, ${ }^{2} \mathrm{ACC}$ Frota, ${ }^{3} \mathrm{CC}$ Sant'Anna, ${ }^{4} \mathrm{ES}$ Goudouris, ${ }^{5} \mathrm{RM}$ Maron. ${ }^{1}$ Unidade de Pacientes Internos, Instituto de Puericultura E Pediatria Martagão Gesteira Da Universidade Federal Do Rio de Janeiro, Rio de Janeiro, Brazil; ${ }^{2}$ Infectologia Pediátrica, Instituto de Puericultura E Pediatria Martagão Gesteira Da Universidade Federal Do Rio de Janeiro, Rio de Janeiro, Brazil; ${ }^{3}$ Pneumologia Pediátrica, Instituto de Puericultura E Pediatria Martagão Gesteira Da
Universidade Federal Do Rio de Janeiro, Rio de Janeiro, Brazil; ${ }^{4}$ Alergia e Imunologia Pediátrica, Instituto de Puericultura E Pediatria Martagão Gesteira Da Universidade Federal Do Rio de Janeiro, Rio de Janeiro, Brazil; ${ }^{5}$ Neurocirurgia Pediátrica, Instituto de Puericultura E Pediatria Martagão Gesteira Da Universidade Federal Do Rio de Janeiro, Rio de Janeiro, Brazil

\subsection{6/archdischild-2014-307384.880}

Background and aim Tuberculosis is a highly prevalent disease in Brazil, but cranial bone presentation are extremely rare. We describe a case report of atypical cranial bone tuberculosis in a tertiary care teaching hospital in Rio de Janeiro, Brazil.

Methods PHGR, 4 years age, male, caucasian, born and raised in Saquarema, RJ, Brazil. The patient was admitted in September 2013 with back pain, fever, spleen and liver nodular lesions. Multiple osteolytic lesions were also found, more importantly in C6, C7 and cranial bone. A hepatic biopsy showed granulomatous hepatitis. Due to the cervical severity lesions, an empiric treatment for tuberculosis with RIP (Rifampicine, Isoniaside and Pirazinamide) began. Sixty days after treatment progressive improvement of all osteolytic lesions except on the cranial bone and calcified spleen and liver granulomas were observed. A cranial bone biopsy showed new granulomas and BAAR were visualised. After 5 months of RIP treatment the cranial bone lesions began to fade. He had not received the BCG vaccine (Calmet-Guerrin Baccilus) despite of this being part of the official brazilian immunisation schedule. The child has also been evaluated for Mendelian susceptibility to mycobacterial diseases. Results We present a case of multisystemic tuberculosis in a boy with cranial bone compromising.

Conclusions Widespread tuberculosis can be seen in children that do not receive the BCG vaccine.

\section{PO-0229 CONGENITAL TOXOPLASMOSIS (CT) IN INFANTS FOLLOWING IN VITRO FERTILISATION WITH IMMUNOSUPPRESSION IN EARLY PREGNANCY, TWO CASE REPORTS}

${ }^{1}$ AM Deasy, ${ }^{1}$ SMC Slattery, ${ }^{2}$ W Ferguson, ${ }^{1} \mathrm{P}$ Gavin, ${ }^{1} \mathrm{KM}$ Butler. ${ }^{1}$ Paediatric Infectious Diseases, Our Ladies Childrens Hospital Crumlin, Dublin, Ireland; ${ }^{2}$ Infectious Diseases, The Rotunda Hospital, Dublin, Ireland

\subsection{6/archdischild-2014-307384.881}

Introduction In Ireland, 75\% of pregnant women are seronegative for toxoplasma, making them susceptible to primary infection during pregnancy.

First case: A female infant was conceived by IVF. Her mother received high dose steroids, humira and intravenous immunoglobulin at the initiation of, and during early pregnancy. The infant was neurologically abnormal at birth, had marked ventricular dilatation, intracranial calcification and bilateral retinal detachments. CT was confirmed with infant toxoplasma IgG and IgM positive. Maternal serology was consistent with primary maternal infection during pregnancy. Despite anti-toxoplasma therapy, the infant succumbed at six months of age.

Second Case: A one-year-old girl was investigated for a convergent strabismus. Conceived by IVF, her mother received high dose steroids for the first four months of pregnancy. Developmentally normal, at nine months of age she developed a right strabismus. Dilated fundoscopy revealed an extensive right macular scar. Neuroimaging showed intracranial calcification. Although toxoplasma IgM was negative, CT was diagnosed based on strongly persistent Toxoplasma IgG Ab, without evidence of 